\section{GD Reviews}

\section{P. Kuljuntausta: Momentum \\ Robert Denham}

Can a work of sound art (music) survive on color alone? Petri Kuljuntausta makes a good case for it in his most recent CD, Momentum. All of the work represented on his program rely heavily on the concept of color as subject matter, some exclusively so. Kuljuntausta's fascination with color takes form in keen interest in the building blocks of sound and how these various elements (harmonics, beating patterns, etc.) can be isolated and magnified so that they begin to function as their own entities apart from the sonorities within which they originated. As a result, his music is more concerned with the steady revelation of these hidden components than with any sort of linear form or presentation of themes, which would be indicative of the classical tradition. With this in mind, one should not listen to Kuljuntausta's music with the expectation that it should methodically progress from one point to another, but should instead revel in the experience of being enlightened as to the latent powers of expression that are contained within "simple" particles of sound.
Canvas (1999) relies almost exclusively on the preoccupation with color described above. The piece begins with a sample of male voices singing an $\mathrm{F}$ major chord. Kuljuntausta stretches this sample to the point that it carries a certain timeless quality, and eventually creates other chords that are closely related to the original $\mathrm{F}$ major (D minor, for instance). Within this slow-moving texture, he alternately magnifies specific pitches or overtones that occur naturally within these chords. The overall sense of this music is that it is derived from Medieval practice, emulating in some distant way the straight tones, smooth swells, and cathedral quality decays of Gregorian Chant. This piece does reveal a subtle sense of progression the male voices, which begin with "Ah" tones, gradually evolve to express some Latin texts), though it is primarily concerned with exposing the variety of different color possibilities within the original sample.

Violin Tone Orchestra (1996) betrays the influence of Steve Reich, as Kuljuntausta momentarily sets aside the concept of "color as king" in favor of exposing the rhythmic possibilities within a given sample. Just as Reich explored the possibilities of phase shifting in his early tape-loop pieces (Come Out, It's Gonna Rain), Kuljuntausta takes a short sample from one of his own string quartets and phase shifts it against itself. Sometimes the phasing takes place at such a slow rate that it is barely perceivable; at other times, it is abrupt and the changes are immediately apparent. The process is still typical of Kuljuntausta's interest in finding the profound within the mundane, since he is exploring the rich possibilities contained within a seemingly insignificant fragment of sound.

The title of the third piece on the disc, Four Notes (2001), is not surprising considering the composer's tendency to use limited resources to produce maximum results. This piece is also derived from samples of the string quartets mentioned above, and is similar to Canvas in that it sets up a relatively static texture out of which pinpoints of color are explored under a musical microscope. This piece, by virtue of its lack of direction, makes it obvious that Kuljuntausta does not consider horizontal form as being an essential element in the message he is trying to convey.

Just as the focus on color in Canvas is reflected by similar practices in Four Notes, so the concept of phase shifting within Violin Tone Orchestra is reflected in the fourth piece, When I am Laid in Earth (2002). In the latter, Kuljuntausta works with a limited sound palette (again he is using samples of his string quartets, along with synthetically produced harpsichord sound), and creates rhythmic friction between a pair of two-note figures. These figures are set in opposition to one another so that the first pattern is slightly slower than the second. As a result, periods where the figures seem to correspond alternate with moments where the patterns are obviously disjunct from one another. In addition, the individual figures themselves eventually split to produce a total of two string and two harpsichord samples.

Freedom (1998) is yet another work that gives color the central role, but it is unique among the other pieces on this $\mathrm{CD}$ in that it focuses less on isolated overtones and more on the beating produced by the harmonic relationships between various pitches. A low pedal $\mathrm{A}$ is established near the beginning as a sort of ominous drone from which the texture is not allowed to escape until the end, when it finally steps up to B. As other pitches are set against this pedal, beatings result in the pedal itself so that it "grumbles." This is yet another work where Kuljuntausta creates a sense of timelessness due to a lack of harmonic progression; one gets the impression that the composer intends these pieces to be seen as "meditative escapes," where a given concept is evident from the start and has only to be experienced (less thinking, more feeling). In this sense, it is not so important that the listener be present for every moment of the piece, though doing so would allow the full meditative experience that Kuljuntausta envisions.

Counterpoint plays a major role within Momentum, which suggests a trend on the 
part of Kuljuntausta towards referencing music of the past (remembering his reference to the medieval period in Canvas). In the course of this piece, string samples are contrasted with an electric guitar patch, and these timbres are subtly altered to produce vague shades of color, or ghostlike effects. As one might suspect from the work's title and its contrapuntal texture, a subtle sense of direction does present itself in the form of a gradual shift from simple two-voice counterpoint to a more complex web of contrapuntal lines. In this way, Momentum stands apart from its peers by virtue of taking more care in the process of "getting from here to there."

In the Beginning (2001) is a provocative reference to the first chapter of Genesis. Again, the composer uses string quartet samples, but this time they are presented as a slow-moving backdrop against which other sampled sounds are set. These samples include city noises such as traffic and trains, coupled with natural sounds such as birds and wind. The composer is still interested in the integral components of the string quartet sample, magnifying particular harmonics or other pitches, but these are not so invasive as in the other pieces. The quartet samples are subservient to the other sampled sounds, much like the string ensemble in Ives's Unanswered Question is a backdrop against which the proverbial question is asked. As may be expected, In the Beginning moves at an incredibly slow rate of speed (if it can be said to be moving at all); listening to this piece is like sitting in Golden Gate Park on a sunny afternoon, experiencing the curious conflict between the urban and natural worlds.

Kuljuntausta does not present the listener with an endless variety of stimuli, but chooses instead to focus on the variety that is contained (though often overlooked) within limited sources. His music offers the listener an opportunity to explore the many possibilities inherent to these sources, and to briefly step back and appreciate the beauty of "common" sound. In this light, his $\mathrm{CD}$ is a great success, and deserves the consideration of every serious appreciator of computer music.

\section{Rodrigo Sigal, Space Within} Jennifer Bernard Merkowitz

Rodrigo Sigal's new solo CD, Space Within, is a tour de force of complex yet clearly defined aural interaction. The music, all written between 1999 and 2002 while the composer was working on his $\mathrm{PhD}$ at City University in London, focuses on the relationships between sound worlds, whether they are synergistic or fraught with tension. Sigal's goal was to "generate emotions by exploring the relationships between human beings and computer generated sound material," and the pieces were part of a thesis entitled "Compositional Strategies in Electroacoustic Music." As such, each piece demonstrates a different strategy and dynamic between acoustic instrument and electronics (or, in the case of the two fixed format pieces, between different recorded materials), and Sigal is a master of managing those subtle variations. Coupled with remarkable performances by three different instrumentalists, the execution of his ideas is coherent, musical, and memorable.

Sigal, who was born in Mexico in 1971 , demands much of his listener: he states that his music "cannot be understood while the listener is engaged in other activities," and recommends the use of headphones or a high-quality sound system. Indeed, his music requires fixed concentration in order to appreciate all the subtle changes in timbre, clever spatial effects, and carefully planned structures. The experience of listening to this $\mathrm{CD}$ with headphones is phenomenal; I highly recommend it.

The first piece on the $\mathrm{CD}$, and my favorite is Friction of Things in Other Places (2002). It focuses on the musical ideas that can stem from disparate sounds happening simultaneously. The piece starts out with the peaceful sound of wind chimes, and traverses parallel soundscapes where we hear voices, electronic hum, water, coins, and the heavy drums of pop music before returning to the sounds of the beginning. It is almost as if the listener is taken on a journey through space at one moment in time, and then suddenly gets yanked backwards to the point of departure. Each transition is beautifully handled, and the connections between the diverse sounds seem both jarring and completely logical at the same time. Sigal takes care to manage his themes so that the listener can follow the trajectory of the piece while still exploring variations and relationships with other sounds.

Rimbarimba (Lejos Del Silencio) (2002) for marimba and Max/MSP-triggered electroacoustic sounds is the next piece on the disc, opening with a memorable octave riff accented by an electroacoustic whooshthud (pardon the onomatopoeia). In this piece, Sigal visualizes the human performer as a "bridge" between the artificial sound worlds and the recorded sounds related to the marimba. The marimba and the electroacoustic sounds draw from each other, and their relationship is made evident in the connections between their rhythmic materials and their spectral characters. The electronic sounds turn the marimba into a kind of "metainstrument" by adding to the timbre of the live performer's line. At times, this enhancement is very subtle, like the slight detuning of the marimba's pitches, and at other times the electronics add another element to the sound, making for a duet 
that is so tightly integrated it still somehow sounds like one instrument. Robert Esler performs this piece with great nuance and skill, and seems very conscious of his role as the bridge, blending in seamlessly with the electronics. The only thing that bothered me about this piece was the ending; a female voice enters very close to the end, breaking through the sound world of the past fourteen minutes and triggering a flourishing close in the marimba. While the ending was convincing, I found myself wondering how the voice was connected to the rest of the piece.

Track 3 is Twilight (2000), a piece for bassoon and electroacoustic sounds. It consists of four continuous movements based on the four types of twilight: civil, nautical, astronomical and true night. For those unfamiliar with this concept (as I was before looking it up), here is a brief explanation: before the sun rises and after it sets, civil twilight occurs when the sun is up to 6 degrees below the horizon, nautical twilight occurs when the sun is from 6 to 12 degrees below the horizon, astronomical twilight is when the sun is 12 to 18 degrees below, and beyond that is true night. The four stages delineate how much can be seen and distinguished at various points of the sun's rising and setting. This concept is an integral part of Treilight, which tries "to evoke the daily and almost imperceptible process of light fading away and night covering the sky" by drawing a parallel between sunlight and sound. The blurring of sight as the world becomes dark is made manifest in aural illusions; there are many moments when the listener is unsure which line is being performed live and which sounds are electronic trickery. As the piece progresses, the brightness and clarity of the electronic sounds fade, and they evolve from eerie meta-bassoon glissandi and extremely high-pitched nasal riffs to percussive thumps, glitches, and sounds of wind, to simple resonating timbres growing out of the live bassoon's long notes. The bassoonist, Wendy Holdaway, does a fabulous job of manipulating the sound of her instrument to reflect these changes in her "environment." Just as the sun's light over the horizon slowly fades away, the piece ends with a very "dark-sounding" fadeout - a fitting conclusion to Sigal's creative version of musical twilight.

The next piece on the CD is Cycles (1999), which is in two movements that are composed similarly: "Stop" and "potS." Sigal describes the concept as "Feelings and sounds coming back to our memory. 'Cycles' of ideas, transformations, processes, and sounds." Both movements use similar source material - a voice saying "stop," and a dichotomy between highand low-pitched sounds. However, they come off very differently because of the way the material is treated. "Stop," the longer of the two, has a more relaxed and logical trajectory. A lone high tone begins the piece, and gradually becomes more complex in timbre. It is joined by lower swells and thuds, and the two lines become intertwined to introduce the spoken voice. The rest of the piece builds from this type of interaction. "Memories" of sounds past weave in and out of the texture. Eventually, the voice transforms and becomes part of the previously separate registers. "Stop" is an excellent example of Sigal's mastery of creating strategic relationships between sound worlds.

The second movement, "potS," has a faster pace and more tension. It is clear that this movement has the same concept and source sounds as the previous one; however, the vocal sounds appear more in distress, and the sounds have a more synthetic quality than those of the first movement. Clearly, the feelings and sounds coming back to our memory in this movement are not as pleasant. This movement also seems less focused. As a whole, though, the two movements together create a very interesting demonstration of composing out the same idea in two different ways. I'm not sure that one without the other would work as well. By composing two movements, Sigal introduces another layer of relationships: that of a sound to its counterparts in the other movement.

Tolerance (2000), for cello and electroacoustic sounds, closes the disc on Track 6. As the title suggests, the relationship between the live instrument and the electronics in this piece is one of peaceful coexistence. Both take turns in the spotlight, and it is as if they are having a conversation, "translating" gestures from cello to electroacoustic sounds and vice versa. The cello has some very lovely lines, and Thomas Gardner makes the instrument sing while flawlessly executing difficult double-stops and harmonics. About halfway through the piece, strangely reverbed voices enter into the electroacoustic vocabulary. This makes the piece a little creepy for a couple minutes, and I was relieved when the voices went away. The rhythmic activity gradually loses speed until the piece ends in a slow counterpoint of cello harmonics and high-pitched electroacoustic responses. It was a very beautiful finish for the CD.

On the whole, I would highly recommend the music on Rodrigo Sigal's Space Within. Sigal composes with a fresh awareness of the possibilities and relationships inherent in electroacoustic music, and he is not afraid to make bold juxtapositions and craft them into convincing musical ideas. He has a lyric sensibility and a keen sense of structure that combine to make his pieces clear, enjoyable, and memorable. We can only wait for more great music to come from this promising young composer. 